\title{
Research Concerning the Correlations Between Some Experimental Results in the Case of a Sucker Rod Pumping Installation
}

\begin{abstract}
DORIN BADOIU, GEORGETA TOMA*
Petroleum-Gas University of Ploiesti, 39 Bucuresti Blvd., 100680, Ploiesti, Romania

In the paper are analyzed the correlations between the experimental results obtained for the instantaneous rotation speed of the cranks shaft of a conventional sucker rod pumping installation and the speed and the acceleration at the end of the polished rod. The correlations have been established by analyzing the kinematics of the mechanism of the sucker rod pumping unit. The experimental records have been processed with the program Total Well Management. A computer program for performing the simulations has been developed by the authors using Maple programming environment.
\end{abstract}

Keywords: pumping unit, kinematics, cranks shaft, polished rod

The data collected by the transducers ( of load, position, pressure, power etc) with which are equipped the sucker rod pumping installations provides a lot of information concerning the pumping system's behavior during functioning [1-3]. The information from some of these transducers gives an overview of the operation of the pumping system, such as: the variation of the motor power, the variation of the gearbox torque and the variation of the instantaneous rotation speed of the cranks shaft, while other transducers provides information concerning the kinematics and the dynamics of the sucker rod column (for example: the variation of the position, speed and acceleration of the polished rod and the surface dynagraph) and the functioning of the depth pump, such as: the variation of the plunger position and of the instantaneous pump displacement. The correlations between the data acquired by these transducers are particularly useful for development or verification of different models concerning the kinematics and the dynamics of the pumping installations $[4,5]$.

In this paper are analyzed the correlations between the experimental results obtained at a well serviced by a C640D-305-120 pumping unit for the instantaneous rotation speed of the cranks shaft and the speed and the acceleration at the end of the polished rod. For this purpose is analyzed the kinematics of the mechanism of the sucker rod pumping unit. The experimental records have been processed with the program Total Well Management [6]. For simulations a computer program has been developed by the authors using Maple programming environment [7] .

\section{Experimental part}

The experimental records have been processed with the program Total Well Management [6]. It was analyzed a well serviced by a C-640D-305-120 pumping unit produced by Lufkin [8].

For establishing the variation on a cinematic cycle of the speed and of the acceleration at the end of the polished rod using the simulation program mentioned before were used the records concerning the variation of the instantaneous angular speed of the cranks shaft for two strokes: stroke 10 (fig.1) and stroke 25 (fig. 2). The instantaneous angular speed of the cranks shaft is expressed in strokes per minute (SPM) what it is equivalent to rotations per minute.

The simulation results have been compared with the records of the variation of the speed and of the acceleration

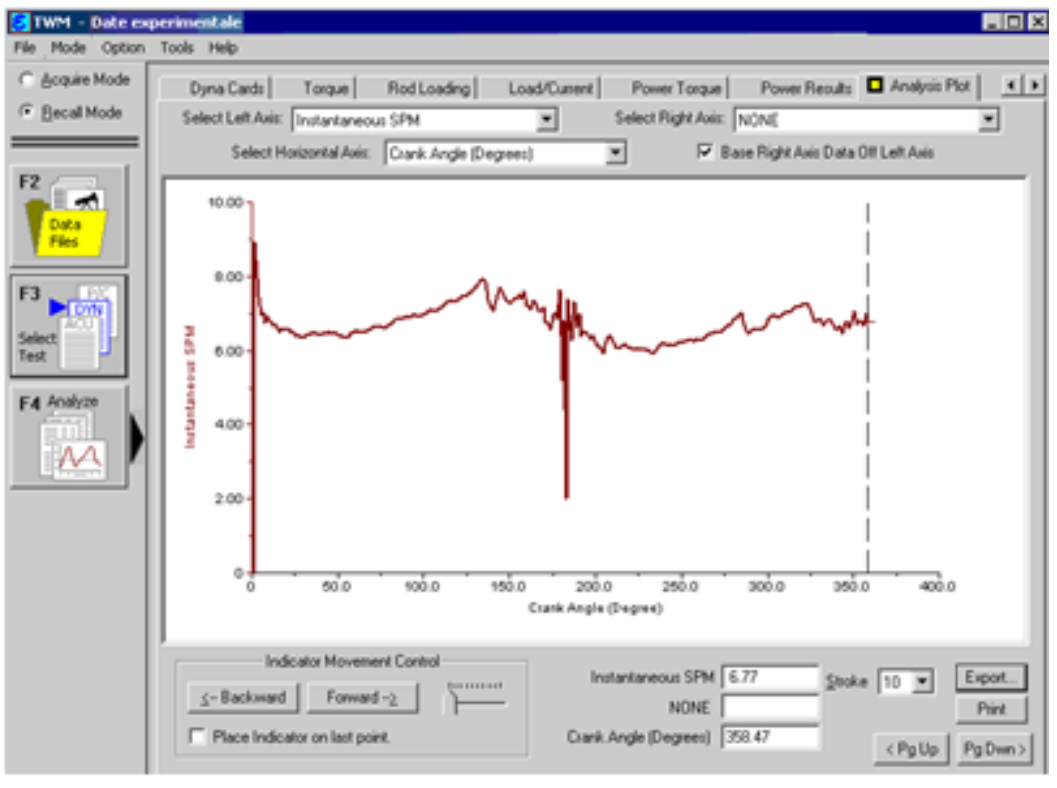

Fig. 1. The variation of the instantaneous rotation speed of the cranks shaft during the stroke 10

* email: georgeta_tm@yahoo.com 

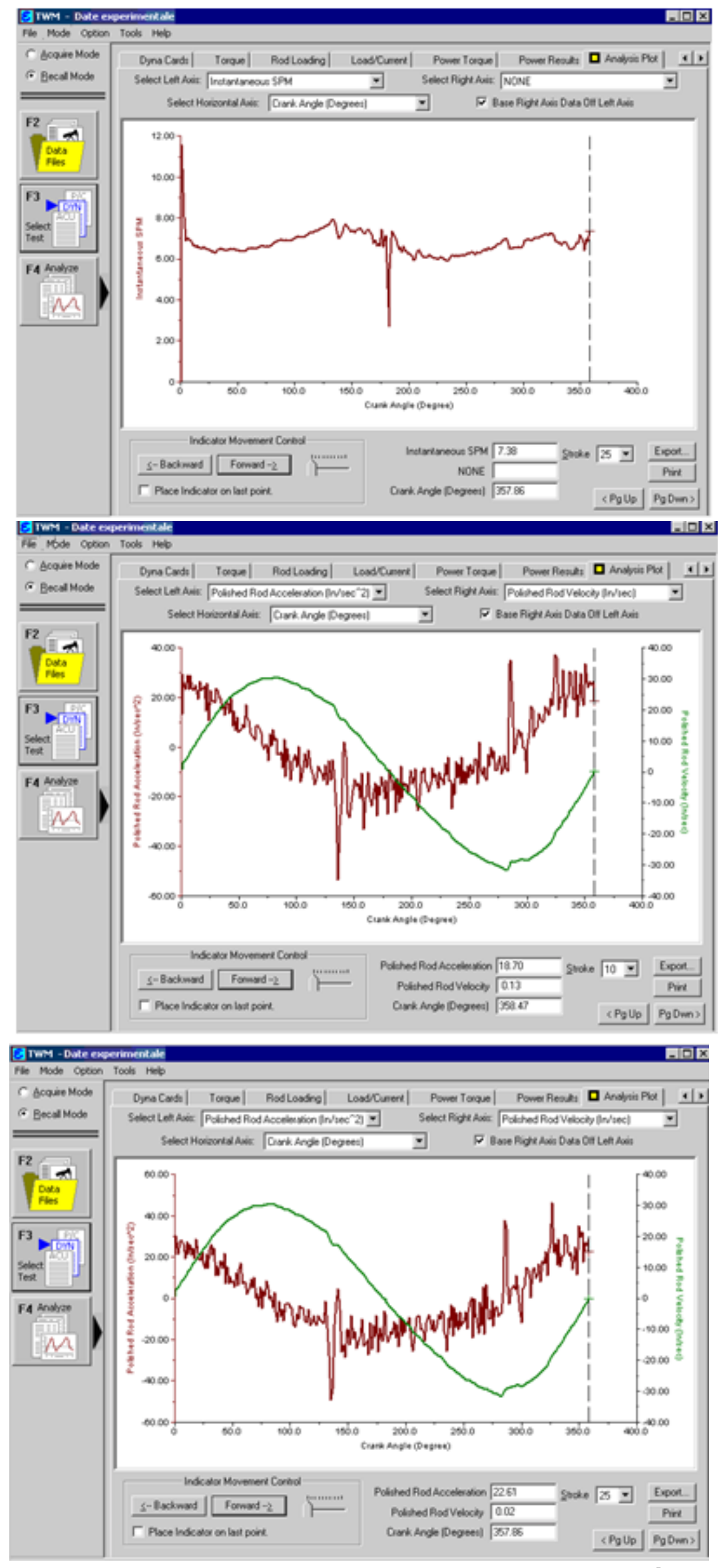

at the end of the polished rod for the two strokes (fig. 3 and fig. 4).

\section{Cinematic analysis of the mechanism of the conventional sucker rod pumping units}

The mechanism of the conventional pumping units is presented in figure 5 . With 1 were noted the cranks of the
Fig. 2. The variation of the instantaneous rotation speed of the cranks shaft during the stroke 25

Fig. 3. The variation of the speed and of the acceleration at the end of the polished rod during the stroke 10
Fig. 4. The variation of the speed and of the acceleration at the end of the polished rod during the stroke 25 


$$
\omega_{3}=\dot{\varphi}_{3}=\frac{d \varphi_{3}}{d \varphi_{1}} \cdot \frac{d \varphi_{1}}{d t}=\omega_{1} \cdot \frac{d \varphi_{3}}{d \varphi_{1}}=\frac{\pi \cdot n_{1}}{30} \cdot \frac{d \varphi_{3}}{d \varphi_{1}}
$$

where $n_{1}$ is the angular speed of the cranks expressed in rotations per minute.

The variation of the angle $\varphi_{3}$ (fig. 5) according to the angle $\varphi_{1}$ can be determined from the following system of equations obtained by projecting on $O x$ and $O y$ axes the vector equation $\overline{O A}+\overline{A B}+\overline{B C}=\overline{O C}$

$$
\left\{\begin{array}{l}
O A \cdot \cos \varphi_{1}+A B \cdot \cos \varphi_{2}+B C \cdot \cos \varphi_{3}=x_{C} \\
O A \cdot \sin \varphi_{1}+A B \cdot \sin \varphi_{2}+B C \cdot \sin \varphi_{3}=y_{C}
\end{array}\right.
$$
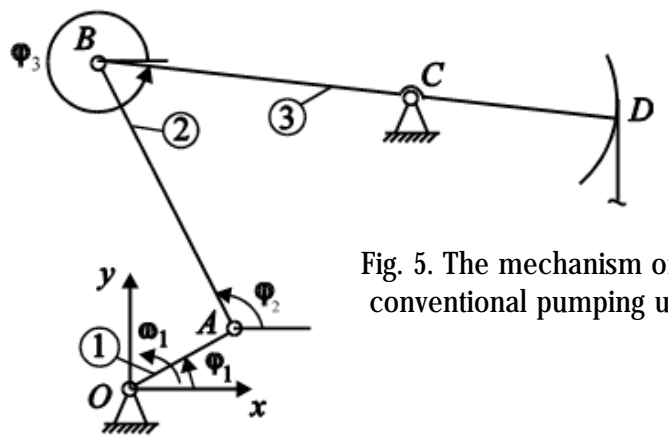

Fig. 5. The mechanism of the conventional pumping units

By rewriting the system of equations (2) into the form:

$$
\left\{\begin{array}{l}
O A \cdot \cos \varphi_{1}+B C \cdot \cos \varphi_{3}-x_{C}=-A B \cdot \cos \varphi_{2} \\
O A \cdot \sin \varphi_{1}+B C \cdot \sin \varphi_{3}-y_{C}=-A B \cdot \sin \varphi_{2}
\end{array}\right.
$$

and by squaring the two equations from (3) and then summing them, it results the following equation from which can be calculated the angle $\varphi_{3}$ :

where:

$$
A_{3} \cdot \sin \varphi_{3}+B_{3} \cdot \cos \varphi_{3}=C_{3}
$$

$$
\left\{\begin{array}{l}
A_{3}=2 \cdot O A \cdot B C \cdot \sin \varphi_{1}-2 \cdot B C \cdot y_{C} \\
B_{3}=2 \cdot O A \cdot B C \cdot \cos \varphi_{1}-2 \cdot B C \cdot x_{C} \\
C_{3}=A B^{2}-O A^{2}-B C^{2}-x_{C}^{2}-y_{C}^{2}+2 \cdot x_{C} \cdot O A \cdot \cos \varphi_{1}+2 \cdot y_{C} \cdot O A \cdot \sin \varphi_{1}
\end{array}\right.
$$

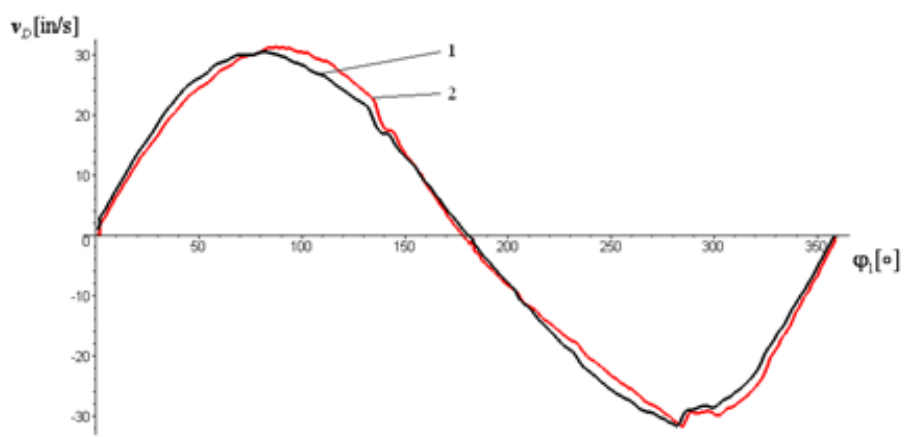

Fig. 6. The variation of the speed at the end of the polished rod for the stroke 10 (experimental - curve 1; simulation - curve 2)
The acceleration $a_{0}$ at the end of the polished rod can be established by deriving with respect to time the speed $v_{D}$ by taking into account the equation (1):

$$
a_{D}=\dot{v}_{D}=C D \cdot \frac{\mathrm{d}^{2} \varphi_{3}}{\mathrm{~d} \varphi_{1}^{2}} \cdot\left(\frac{\pi \cdot n_{1}}{30}\right)^{2}+C D \cdot \frac{\mathrm{d} \varphi_{3}}{\mathrm{~d} \varphi_{1}} \cdot n_{1} \cdot \frac{\mathrm{d} n_{1}}{\mathrm{~d} \varphi_{1}} \cdot\left(\frac{\pi}{30}\right)^{2} \text { (6) }
$$

\section{Results and discussions}

The cinematic analysis of the mechanism of the conventional sucker rod pumping units presented before has been transposed by the authors into a computer program using Maple programming environment [7]. The simulations have been performed in the case of a C-640D305-120 pumping unit produced by Lufkin [8], whose component elements have the following dimensions: $O A$ $=30$ in $(0.762 \mathrm{~m}) ; A B=133.5 \mathrm{in} .(3.3909 \mathrm{~m}) ; B C=111.09$ in. $(2.8217 \mathrm{~m}) ; C D=155 \mathrm{in} .(3.937 \mathrm{~m})$. The coordinates of the point $C$ (fig. 5) are [8]: (2.8194 m) and (3.5052 m).

In figures $6 \div 9$ are presented the variations of the values of the speed $v$ and of the acceleration $a_{0}$ at the end of the polished rod for the strokes 10 and 25 , respectively. The curves 1 represent the experimental results and the curves 2 correspond to the simulation results. For obtaining the simulation results have been used the records concerning the variation of the instantaneous angular speed of the cranks for the two strokes presented in figures 1 and 2 .

Figures $6 \div 9$ highlight a very good accordance between the experimental and simulation results, especially regarding the variation of the acceleration at the end of the polished rod. From figures 8 and 9 can be observed that the simulation results regarding the acceleration at the end of the polished rod practically track all the small variations of the experimental values of the acceleration.

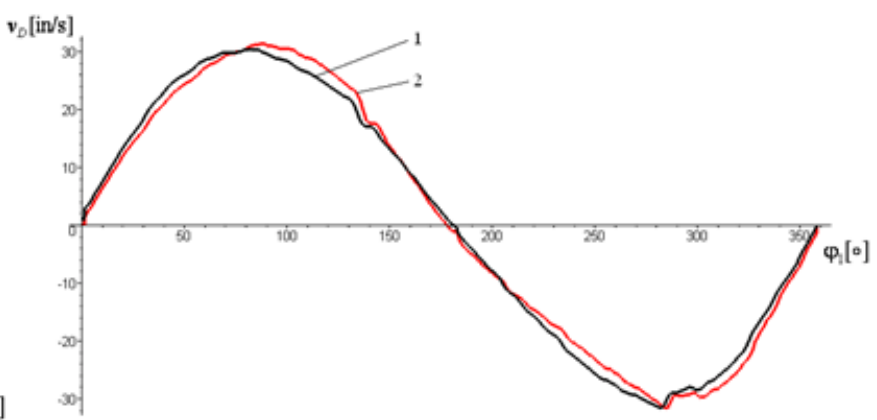

Fig. 7. The variation of the speed at the end of the polished rod for the stroke 25 (experimental - curve 1; simulation - curve 2)

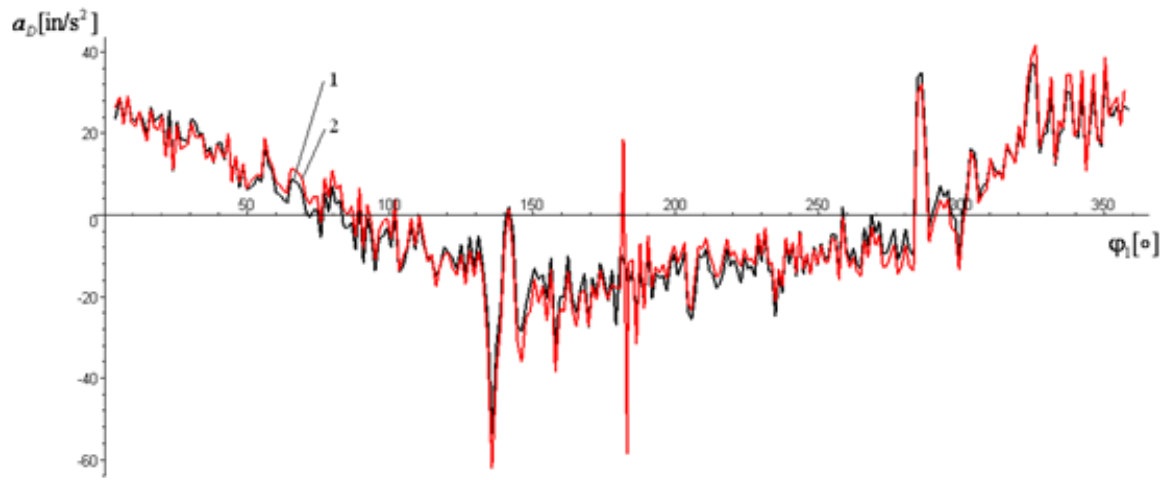

Fig. 8. The variation of the acceleration at the end of the polished rod for the stroke 10 (experimental - curve 1; simulation -curve 2) 


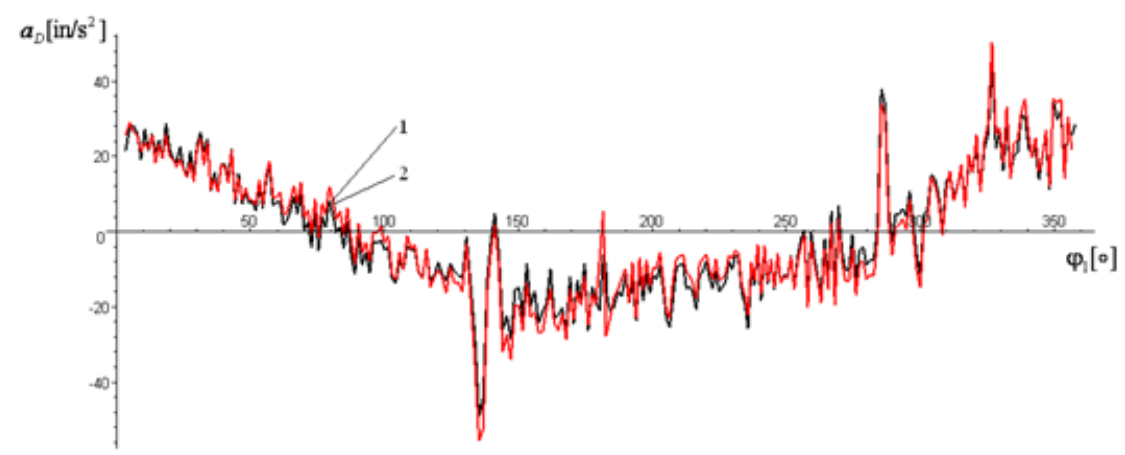

Fig. 9. The variation of the acceleration at the end of the polished rod for the stroke 25 (experimental - curve 1 ; simulation - curve 2)

\section{Conclusions}

In this paper has been presented some results concerning the cinematic analysis of the mechanism of the conventional sucker rod pumping units, highlighting the manner of determining the speed and the acceleration at the end of the polished rod. It has been analyzed in this way the correlations between the experimental results obtained at a well serviced by a C-640D-305-120 pumping unitfor the instantaneous rotation speed of the cranks shaft and the speed and the acceleration at the end of the polished rod. For processing the experimental records has been used the program Total Well Management and for simulations has been developed a computer program using Maple programming environment. It has been obtained a very good accordance between the experimental and simulation results, especially regarding the variation of the acceleration at the end of the polished rod.

\section{References}

1.POPOVICl, A., Equipment for the exploitation of oil wells (in Romanian), Technical Publishing House, Bucharest, 1989
2.GIBBS, S G., Predicting the behavior of sucker-rod pumping systems, Journal of Petroleum Technology, 1963 (July), p. 769-778

3.GIBBS, S G., Computing gearbox torque and motor loading for beam pumping units with consideration of inertia effects, Journal of Petroleum Technology, 1975 (September), p. 1153-1159

4.BADOIU, D., TOMA, G., Research concerning the identification of some parameters of a sucker rod pumping unit, Rev. Chim. (Bucharest), 68, no. 10, 2017, p. 2289-2292

5.TOMA, G., PUPAZESCU, A., BADOIU, D., On the kinematics of some sucker rod pumping units, Petroleum-Gas University of Ploiesti Bulletin, Technical Series, Vol. LXVI, No. 3, 2014, p. 95-100

6.***, Total Well Management Help Manual, Echometer Company, Texas

7.MONAGAN, M.B., GEDDES, K.O., HEAL, K.M., LABAHN, G., VORKOETTER, S.M., MCCARRON, J , DEMARCO, P., Maple Introductory Programming Guide, Maplesoft, a division of Waterloo Maple Inc., 2005

8.***, Conventional Crank Balanced Pumping Units, Lufkin Oilfield Products Group, Houston, Texas

9.BADOIU, D., Structural and cinematic analysis of the mechanisms (in Romanian), Technical Publishing House, Bucharest, 2001

$\overline{\text { Manuscript received: } 31.01 .2018}$ 